\title{
Which is the most appropriate strategy for conducting multivariate voxel-based group studies on diffusion tensors?
}

\author{
Alix Bouchon ${ }^{\mathrm{a}, *}$, Vincent Noblet $^{\mathrm{a}}$, Fabrice Heitz ${ }^{\mathrm{a}}$, Julien Lamy ${ }^{\mathrm{a}}$, Frédéric \\ Blanc $^{\mathrm{a}, \mathrm{b}}$, Jean-Paul Armspach ${ }^{\mathrm{a}}$ \\ ${ }^{a}$ ICube, University of Strasbourg, CNRS, Fédération de Médecine Translationnelle de \\ Strasbourg (FMTS), France \\ ${ }^{b}$ Geriatry service, Memory Resources and Research Center (CMRR), University Hospital of \\ Strasbourg, France
}

\begin{abstract}
There is a real need in the neuroscience community for efficient tools to compare Diffusion Tensor Magnetic Resonance Imaging across cohorts of subjects. Most studies focus on the comparison of scalar images such as fractional anisotropy or mean diffusivity. Although different statistical frameworks have been proposed to compare the whole diffusion tensor information, they are still seldom used in neuroimaging studies. In this paper, we investigate on both simulated and real data whether there is a real added value of considering the whole tensor information for conducting voxel-based group studies. Then, we compare two statistical tests dedicated to tensor, namely the Cramér test and a tensorbased extension of the General Linear Model (GLM), the latter presenting the advantage to account for covariates. We also evaluate the impact of different metrics (Euclidean, Log-Euclidean and affine-invariant Riemannian metrics) for estimating the GLM parameters. Finally, we address the problem of interpreting the change detection maps obtained by tensor-based methods by proposing a way to characterize each of the detected clusters according to several scalar indices. Our study suggests that if there is no prior assumption about the nature of the expected changes, it is really preferable to use tensor-based rather
\end{abstract}

\footnotetext{
${ }^{*}$ Corresponding author

Email address: a.bouchon@unistra.fr (Alix Bouchon)
} 
than scalar-based statistical analysis. The Cramr test can advantageously be used when no confounding variable hampers the group comparison, otherwise the GLM should be considered. Finally, the different metrics show similar performance in the real scenario, with a significant computational overhead for the Riemannian framework.

Keywords: Diffusion tensor imaging, general linear model, group comparison, tensor-based regression, Euclidean, Log-Euclidean and Riemannian manifolds.

\section{Introduction}

Diffusion Tensor Magnetic Resonance Imaging (DT-MRI) allows clinicians and neuroscientists to investigate the white matter structure by probing water molecule diffusion. Group studies allow to find out brain regions that are statistically different between two populations or that are significantly correlated with some covariates. As a consequence, there is an increasing need for efficient tools to compare DT-MRI across cohorts of subjects according to clinical or cognitive data. This may help to characterize the damages caused by a pathology and to understand the mechanisms underpinning the disease. Group comparisons of DT-MR images are usually performed using either region-of-interest-based analysis, tract-based analysis, or voxel-based analysis [1]. In this paper, we will focus on voxel-based analysis, whose major interest is that it does not make any assumption on the spatial location of the expected changes.

Most voxel-based studies focus on the comparison of scalar images derived from DT-MRI such as Fractional Anisotropy (FA) or Mean Diffusivity (MD) using either the voxel-based statistical analysis framework provided in $\operatorname{SPM}^{1}[2]$ or the tract-based spatial statistics (TBSS) method provided in the FSL library ${ }^{2}[3$. However, these methods do not exploit all the information contained in tensor images and thus cannot detect all kinds of changes. Although different statistical frameworks have been proposed to compare either several scalar

\footnotetext{
$1 /$ http://www.fil.ion.ucl.ac.uk/spm/

2 http://fsl.fmrib.ox.ac.uk/fsl/
} 
indices simultaneously [4, eigenvalues or eigenvectors of diffusion tensors [5], or even the whole diffusion tensor information [6, 7, 8, 9, 10, they remain seldom used in neuroimaging studies.

In this paper, we investigate whether there is a real added value of con25 sidering the whole tensor information as compared to a single scalar index or several scalar indices simultaneously for conducting voxel-based group studies. To this end, we consider a tensor-based extension of the General Linear Model (GLM), which provides a convenient way to carry out statistical analysis while taking into account several covariates. We evaluate the impact of considering different manifolds for estimating GLM parameters, namely the Euclidean, LogEuclidean [11] or the Riemannian framework [12. We also providea comparison with an alternative tensor-based test, namely the tensor-based Cramér test, which has already been reported as the best performer among various tensorbased statistical tests [6]. Finally, we deal with the problem of interpreting the change detection map obtained by tensor-based methods by proposing a way to characterize each of the detected clusters according to several scalar indices. To investigate these different points, a simulation framework has been set up, based on DT-MRI acquisitions of healthy subjects in which different kinds of lesions have been introduced. A group study on a cohort of patients suffering 40 from neuromyelitis optica (NMO) is also presented.

The content of the paper is organized as follows. Section 2 depicts the whole framework for DTI group comparison by describing the pre-processing steps, the spatial normalization, the regression, the statistical analysis and the characterization of the detected clusters. The evaluation framework is presented

45 in section 3 . Results on both simulated and real data are presented in section 4 and discussed in section 5 Finally, conclusions and perspectives are given. 


\section{General framework for DTI group comparison}

\subsection{Overview of the processing pipeline}

The goal of voxel-based group comparison is to decide for each voxel $\mathbf{s} \in \mathbb{R}^{3}$ whether there is a significant difference between two groups of subjects while taking into account some covariates such as age, gender, etc. To this end, all subjects should first be registered into a common space according to a deformable registration method (section 2.2). A prerequisite to this step is to first control the quality of each acquisition to assess whether it can be included in the analysis. This is performed through visual inspection of each DWI image and by using a dedicated tool, DTIPren ${ }^{3}[13$. Then, each Diffusion-Weighted Image (DWI) are corrected for subject motion and eddy current distortions using FSL's Diffusion Toolkit. Finally, tensors are estimated from the DWI images according to a weighted least square (WLS) estimation procedure [14.

After the registration step, all tensor images are filtered in the Log-Euclidean space [11 using a Gaussian kernel (FWHM $=8 \mathrm{~mm}$ in all our experiments). This allows to account for the spatial information from the neighboring voxels and also, by the central limit theorem, to render the data more normally distributed [15]. Then, the statistical analysis is conducted.

A convenient way to carry out statistical analysis while taking into account several covariates is to consider the General Linear Model (GLM). It can be done either on a given scalar image such as FA or MD, or on several scalar images simultaneously, or on tensors directly using either an Euclidean, a Log-Euclidean or a Riemannian metric in the regression (section 2.3). A statistical F-test is then used to evaluate whether a given explanatory variable has a significant contribution in the regression model (section 2.4). Finally, the statistical map is thresholded and each detected cluster is characterized by a signature obtained as a combination of several scalar indices (section 2.5). A graphical overview of the pipeline is presented in Fig. 1 .

3 https://www.nitrc.org/projects/dtiprep/ 


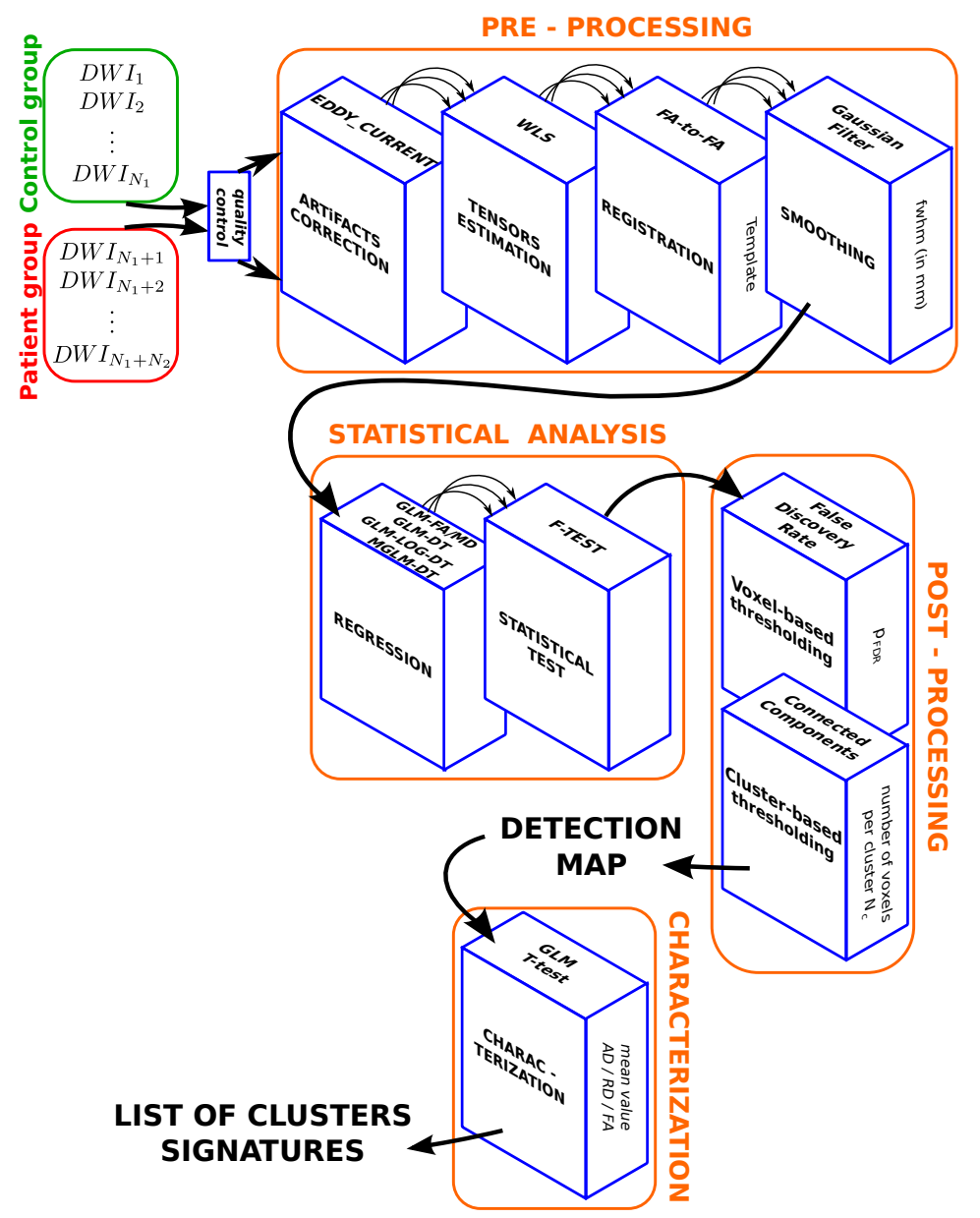

Figure 1: Pipeline overview.

\subsection{Spatial normalization}

Before performing voxel-based statistical analysis, all images are first registered on the same arbitrary chosen reference image belonging to the control group. To this end, all FA images derived from DT-MRI acquisitions are registered through an affine method followed by a non-rigid method [16] using the sum of squared differences metric. Spatial transforms are then applied on tensor images using the Preservation of Principal Direction (PPD) [17] reorientation strategy. This step is required to ensure the orientational consistency of the 
warped tensor field. The basic idea is to apply the local affine component $F$ of the deformation field on the three tensor eigenvectors $\left(e_{1}, e_{2}, e_{3}\right)$, and then to estimate the rotation that leads to the reoriented eigenvectors $\left(\tilde{e}_{1}, \tilde{e}_{2}, \tilde{e}_{3}\right)$, such that $F e_{1}$ and $\tilde{e}_{1}$ are aligned, and such that $F e_{1}$ and $F e_{2}$ span the same plane as $\tilde{e}_{1}$ and $\tilde{e}_{2}$ [17.

\subsection{Multi-linear regression}

In this section, we will present several regression schemes on either scalar images or on tensor fields. Let $\mathcal{M}$ be a manifold and $\left\{y_{i}\right\}_{i \in[1 . . N]} \in \mathcal{M}$ be the observations at a given voxel $\mathbf{s} \in \mathbb{R}^{3}$ from $N$ individuals, each individual being characterized by $K$ explanatory variables $\left\{x_{i, j}\right\}_{j \in[1 . . K]}$ such as age, gender or group affiliation. These observations can be either scalar indices such as FA or $\operatorname{MD}(\mathcal{M} \subset \mathbb{R})$ or full tensors $\left(\mathcal{M} \subset \mathbb{R}^{6}\right)$. The regression problem consists in estimating a function $f: \mathbb{R}^{K} \mapsto \mathcal{M}$ that best fits all the couples $\left(\left\{x_{i, 1} \ldots x_{i, K}\right\}, y_{i}\right)$. A simple parametric approach is to consider the multi-linear function:

$$
y_{i}=\alpha+\beta_{1} x_{i, 1}+\beta_{2} x_{i, 2}+\cdots+\beta_{K} x_{i, K}+\varepsilon_{i}
$$

where $\alpha$ is the intercept, $\beta_{i}$ are the regression coefficients and $\varepsilon_{i}$ are the residuals.

For the scalar case, this corresponds to the standard GLM, which is commonly used in neuroimaging studies [2]. For the tensor case, we investigate the use of different metrics to compute the residuals, namely an Euclidean $\left(\mathcal{M} \subset \mathbb{R}^{6}\right)$, a Log-Euclidean $(\mathcal{M} \subset \operatorname{Sym}(3))$ and a $\operatorname{Riemannian}\left(\mathcal{M} \subset \operatorname{Sym}^{+}(3)\right)$ metric, in order to evaluate their impact in the context of group comparison.

Scalar regression In the scalar case, the General Linear Model (GLM) aims at representing $N$ scalar observations (FA or MD) $Y=\left[y_{1} \ldots y_{i} \ldots y_{N}\right]^{t}$ as a linear combination of $K$ explanatory variables, whose values for the $i^{\text {th }}$ observation are stored in the design matrix $X[i, j]=x_{i, j}$, for $i=1 \ldots N$ and $j=$ $1 \ldots K$. If the residuals $\varepsilon_{i}$ are assumed to be independent and identically distributed (i.i.d.) according to a normal distribution, then the least squares esti- 
mate of $B$ is obtained analytically:

$$
\hat{B}=\arg \min _{B \in \mathbb{R}^{K}}\|Y-X B\|^{2}=\left(X^{t} X\right)^{-1} X^{t} Y
$$

Linear Model for FA (GLM-FA) or for MD (GLM-MD).

Tensor-based regression: Euclidean framework Scalar regression does not capture all the information embedded in the diffusion tensor, in particular the orientation information. Thus, we extended the GLM to take advantage of the full tensor information [10].

A $3 \times 3$ diffusion tensor matrix is symmetric $(\in \operatorname{Sym}(3))$ and may be represented by a vector: $D^{i}=\left[\begin{array}{llllll}D_{x x}^{i} & D_{x y}^{i} & D_{x z}^{i} & D_{y y}^{i} & D_{y z}^{i} & D_{z z}^{i}\end{array}\right]^{t} \in \mathbb{R}^{6}$. The previous regression framework can straightforwardly be extended to the multivariate case by assuming the noise on the tensor components to be i.i.d.. We also assume equal noise variance for all components (homoscedasticity assumption ${ }^{4}$ ). The basic idea is to concatenate all tensor components of the $N$ individuals in a single vector $Y \in \mathbb{R}^{6 N}$. For each explanatory variable, six regressors are estimated, one associated with each tensor component. This is done by constructing a new design matrix $X[i, j]=x_{i, j}$, for $i=1 \ldots N \times 6$ and $j=1 \ldots K \times 6$, where each explanatory variable is replicated in six columns: the first column is composed of the values of the explanatory variable for the entries corresponding to the first tensor components $D_{x x}^{i}$ and of zeros for the others entries, the second column is composed of the values of the explanatory variable for the entries corresponding to the second tensor components $D_{x y}^{i}$ and of zeros for the others entries, and so on for the four others columns. With that formulation, the least squares estimate of $B$ has also a closed-form solution and leads to the estimation of $K$ regressors of six components [10]. This method will be referred to as General Linear Model for Diffusion Tensors (GLM-DT).

\footnotetext{
${ }^{4}$ In a previous work, we have also investigated the heteroscedasticity assumption (i.e., different noise variances for each tensor component), but our experiments did not enable us to exhibit any improvement [10].
} 
Tensor-based regression: Log-Euclidean framework The previous

Euclidean framework does not take into account that diffusion tensors are symetric positive-definite matrices, i.e. $\mathcal{M}=\operatorname{Sym}^{+}(3)$, which is only a subset of $\operatorname{Sym}(3)$. Consequently, the estimated regression can possibly map some sets of explanatory variables to vectors that do not correspond to positive-definite matrices. A way to circumvent this limitation is to conduct the regression on the logarithm of tensors [11. Indeed, the logarithmic transformation enables to map the space of symmetric positive-definite matrices $\mathrm{Sym}^{+}(3)$ to the space of symmetric matrices $\operatorname{Sym}(3)$. Thus, the same previous framework can be used to estimate the regression. The corresponding regression model is then given by:

$$
Y=\exp (X B+\varepsilon)
$$

where $\exp (\cdot)$ stands for the matrix exponential. This method will be referred to as General Linear Model for Diffusion Log-Tensors (GLM-LOG-DT).

Tensor-based regression: Riemannian framework Recently, Kim et al. have proposed a multivariate General Linear Model on Riemannian manifolds, which naturally accounts for the positive-definite nature of diffusion tensors. The corresponding regression model is given by:

$$
y_{i}=\operatorname{Exp}\left(\operatorname{Exp}\left(\alpha, \sum_{j=1}^{K} \beta^{j} x_{i, j}\right), \varepsilon_{i}\right)
$$

where Exp refers to the exponential man The regressors $\beta$ and the intercept $\alpha$ are simultaneously estimated using a gradient descent scheme by minimizing the distance between the data $y_{i}$ and the estimate $\hat{y}_{i}=\operatorname{Exp}\left(\alpha, \sum_{j=1}^{N} \beta^{j} x_{i, j}\right)$. To this end, the following geodesic distance is used:

$$
d\left(y_{i}, \hat{y}_{i}\right)=\sqrt{\left\langle\log \left(y_{i}, \hat{y}_{i}\right), \log \left(y_{i}, \hat{y}_{i}\right)\right\rangle_{y_{i}}}
$$

\footnotetext{
${ }^{5}$ The notation Exp, that refers to the exponential map defined in the context of Riemannian manifolds, should not be confused with the previously used notation exp, that refers to the matrix exponential.
} 
The implementation of this method has been made available online ${ }^{6}$. This method will be referred to as Manifold General Linear Model for Diffusion Tensors $(M G L M-D T)$.

\subsection{Statistical test}

The objective of the statistical test is to evaluate whether a given explanatory variable has a significant contribution in the regression model. To this end, an F-test is used to compare the Residuals Sum of Squares (RSS) of two nested models: a full model taking into account all the covariates $\left(\mathrm{RSS}_{2}\right)$ and a restricted model where the covariate of interest is discarded $\left(\mathrm{RSS}_{1}\right)$ :

$$
F=\frac{\frac{R S S_{1}-R S S_{2}}{p_{2}-p_{1}}}{\frac{R S S_{2}}{N_{o b s}-p_{2}}}
$$

with $p_{2}$ and $p_{1}$ representing respectively the number of covariates for each model and $N_{o b s}$ the total number of data samples?. Assuming that the residuals follow a normal distribution, $F$ follows a Fisher distribution with $p_{2}-p_{1}$ and $N_{o b s}-p_{2}$ degrees of freedom under the null hypothesis that the full model does not provide a significantly better fit than the restricted model. The Gaussianity assumption on the residuals is a reasonable hypothesis for the Euclidean and Log-Euclidean frameworks, but it is no longer valid for the Riemannian framework. In the latter case, permutations may be used to estimate corresponding $p$-values $[9$, but it was not done in this work because of prohibitive computational time. Notice that even for the Euclidean and Log-Euclidean frameworks, alternative p-value estimation strategies can advantageously be used, such as permutations [18] or wild bootstrapping [19] in order to better control Type I errors.

A two-steps post-processing is used to obtain the final detection maps. First, the obtained p-value maps are corrected for the multiple comparisons problem 
using the False Discovery Rate method (FDR) according to a user-defined value (generally $\alpha=0.05$ ). Then, only connected components whose volume is greater than a user-defined threshold $N_{c}$ are kept.

\subsection{Characterization of the detected clusters}

Since tensor-based methods can detect various kinds of changes without providing information on the nature and the direction (in terms of augmentation or diminution) of the observed changes, there is a need to characterize the detected clusters in order to make them interpretable by a physician. Thus, we propose the following procedure to characterize a posteriori the detected clusters in terms of alterations of Axial Diffusion (AD), Radial Diffusion (RD) and Fractional Anisotropy (FA).

It is a three-steps process: first, the spatial means of the three scalar indices $(\mathrm{AD}, \mathrm{RD}$ and $\mathrm{FA})$ are computed on each detected cluster and for each subject. Then, a GLM followed by a two-tailed Student's test are conducted on these values for each scalar index separately, while introducing the same covariates as for the tensor-based statistical analysis. Finally, the list of all the clusters associated with the outputs of the statistical tests for each scalar index is presented to the physician:,+- and n.s. stand respectively for a significant augmentation, a significant diminution and no significative change at a user-defined $p$-value threshold. This may help the physician to interpret each cluster as a favorable or a deleterious change and give him some informations about the nature of the alteration. Notice that this step is performed on the unsmoothed tensor images in order to better reflect the region properties. Indeed, Gaussian smoothing is required to conduct voxelwise analysis, but should generally be avoided for region-based analysis.

We chose to consider $\mathrm{AD}$ and $\mathrm{RD}$, since they have been shown to be valuable biomarkers to characterize induced white matter changes in mouse models [20]. However, using these two indices only is not sufficient to unambiguously interpret all situations, especially when the two indices exhibit significant modifications in the same direction. For instance, an increase (or a decrease) of both 
$\mathrm{AD}$ and $\mathrm{RD}$ can either leave the FA unaltered, or can lead to an augmentation or a diminution of it, depending whether the $\mathrm{AD}$ increases (or decreases) in a higher (or a lower) extent than the RD. Depending on the impact on the FA, the biological interpretation can be very different. This is why, we also consider the FA to disambiguate these cases.

Table 1 provides the list of all possible combinations of AD and RD alterations and their corresponding interpretation. The bottom part of the table details the cases requiring the FA to disambiguate. Notice that clusters that do not present significant alteration of both $\mathrm{AD}$ and $\mathrm{RD}$ are labeled as uncategorisable since it can be explained by either a modification of diffusion orientation or because these clusters present heterogeneous modifications that are averaged out when computing the mean of the scalar indices.

\section{Validation framework}

The evaluation was conducted on both simulated and real data. All DT-MRI images were acquired on a $1.5 \mathrm{~T}$ Siemens scanner with 30 encoding gradients ( $b$ value of $1000 \mathrm{~s} / \mathrm{mm}^{2}$ ) and two baseline images (i.e., $b$-value of $0 \mathrm{~s} / \mathrm{mm}^{2}$ ). The image dimensions are $128 \times 128 \times 41$ and the spatial resolution is $1.8 \times 1.8 \times$ $225 \quad 3.5 \mathrm{~mm}^{3}$.

\subsection{Synthetic data}

The simulated data are built from a group of 22 healthy subjects (23 subjects were initially enrolled, but one examination did not pass the quality control step). A set of 11 images with simulated lesions in the corpus callosum (CC) are generated from the DT-MRI acquisitions of 11 healthy subjects. We focused on the $\mathrm{CC}$ since it consists of a single direction white matter tract, where the second-order tensor assumption holds. These simulated images, considered in the experiments as the patient group, are compared with a group of 11 images of healthy subjects, while introducing age and group affiliation as covariates. For 235 the statistical test, the group affiliation is discarded in the restricted model. Four 


\begin{tabular}{|c|c|c|c|}
\hline $\mathrm{AD}$ & RD & Interpretation of changes & $\begin{array}{l}\text { Type of } \\
\text { changes }\end{array}$ \\
\hline n.s. & + & radial diffusion augmentation & deleterious \\
\hline- & n.s. & longitudinal diffusion diminution & deleterious \\
\hline- & + & $\begin{array}{l}\text { longitudinal diffusion diminution AND } \\
\text { radial diffusion augmentation }\end{array}$ & deleterious \\
\hline n.s. & - & radial diffusion diminution & favorable \\
\hline+ & n.s. & longitudinal diffusion augmentation & favorable \\
\hline+ & - & $\begin{array}{c}\text { longitudinal diffusion augmentation AND } \\
\text { radial diffusion diminution }\end{array}$ & favorable \\
\hline n.s. & n.s. & $\begin{array}{l}\text { diffusion orientation modification OR } \\
\text { cluster with heterogeneous changes }\end{array}$ & uncategorisable \\
\hline
\end{tabular}

\section{Cases requiring the FA to disambiguate}

\begin{tabular}{|c|c|c|c|c|}
\hline $\mathrm{AD}$ & RD & FA & Interpretation of changes & $\begin{array}{l}\text { Type of } \\
\text { changes }\end{array}$ \\
\hline+ & + & n.s. & mean diffusion augmentation & deleterious \\
\hline+ & + & - & radial diffusion augmentation & deleterious \\
\hline+ & + & + & longitudinal diffusion augmentation & favorable \\
\hline- & - & - & longitudinal diffusion diminution & deleterious \\
\hline- & - & n.s. & mean diffusion diminution & favorable \\
\hline- & - & + & radial diffusion diminution & favorable \\
\hline
\end{tabular}

Table 1: List of possible changes of $\mathrm{AD}$ and $\mathrm{RD}$ (and FA if there is an ambiguity) and corresponding interpretation (Legend: $+=$ significant augmentation, $-=$ significant diminution, n. s. = not significant). 


\begin{tabular}{ccc}
\hline Kind of change & Corresponding tensor modification \\
\cline { 1 - 1 } Mean diffusion augmentation & & $\lambda_{i}^{\prime}=(1+\alpha) \lambda_{i}$, with $i=1,2,3$ \\
Radial diffusion augmentation & $\lambda_{i}^{\prime}=\lambda_{i}+\alpha \frac{\lambda_{1}-\lambda_{i}}{2}$, with $i=2,3$ \\
Longitudinal diffusion diminution & $\lambda_{1}^{\prime}=\lambda_{1}-\alpha\left(\lambda_{1}-\lambda_{2}\right)$ \\
Orientation modification & $D^{\prime}=R D R^{t}$, with $R$ a rotation matrix \\
\hline
\end{tabular}

Table 2: Equations to simulate the four kinds of lesions (see 21 for details). $\lambda_{i}$ is the $i^{\text {th }}$ eigenvalue of the tensor matrix sorted in descending order $\left(\lambda_{1}\right.$ represents the eigenvalue associated to the principal direction)

kinds of lesions are simulated: mean and radial diffusion augmentation, longitudinal diffusion diminution and diffusion orientation modification (Table 2).

A parameter $\alpha$ varying from 0 to 0.9 by steps of 0.05 controls the amplitude of the simulated lesions (Fig. 22. An image is generated for each $\alpha$ and each kind of lesions, leading to $4 \times 19=76$ groups of 11 patients images. This allows us to investigate the statistical power of the tests by testing different size effects (through the coefficient $\alpha$ that controls the amount of simulated modification) for a given number of subjects.

Notice that the three first kinds of lesions are consistent with real case scenarios 20]. For instance, a decrease of longitudinal diffusion can be linked to axonal modifications including reduced axonal caliber, while an increase of radial diffusion can be linked to a dysmyelination process. On the other hand, the change of diffusion orientation is rather a toy example, since a realistic diffusion orientation modification would imply the deflection of the whole trajectory of a fiber bundle.

In order to make a fair comparison between all the methods while not introducing a bias by arbitrarily choosing a given p-value estimation procedure, we conducted ROC analysis 22] directly on the computed statistics. This allows us to compare the statistical maps with the ground truth for various statistical thresholds. The Area Under the ROC Curve (AUC) is computed for each experiment. An AUC of $50 \%$ corresponds to a random detection and $100 \%$ to a 


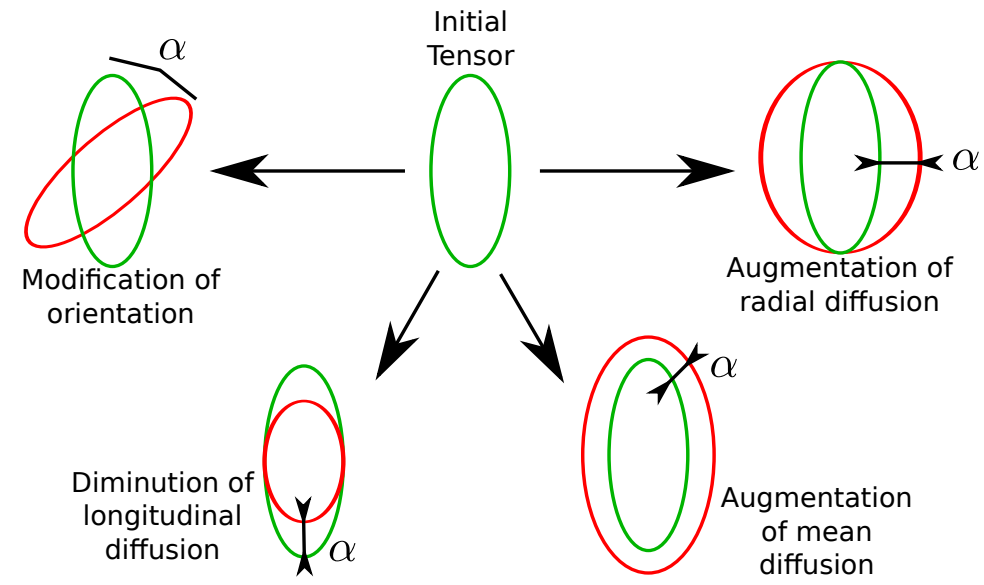

Figure 2: Representation of the four kinds of lesions. The green and the red tensors represent the initial and the modified tensors, respectively.

perfect detection. AUCs are plotted with respect to the modification amplitude $\alpha$ for each kind of simulated lesion (Fig. 3 and 5). By this way, it is possible to assess the performance of each method for major changes as well as smaller ones and for different types of lesions.

\subsection{Neuromyelitis Optica cohort}

The Neuromyelitis Optica (NMO) is an inflammatory disease that mainly affects the spinal cord and the optic nerves. Alterations of the normal appearing white matter have already been demonstrated in several brain areas such as the corpus callosum, the occipital lobe and the cortico spinal tract [23]. These alterations may be associated with clinical disorders such as cognitive, visual (blindness) and motor disorders. Thirty seven NMO patients were initially enrolled. After visual inspection of DWI images, three patients were excluded due to the presence of strong image artifacts. The remaining data successfully passed the quality control performed by the DTIPrep software. Finally, a group of 34 patients suffering from NMO is compared to a group of 22 healthy subjects by considering age and group affiliation as covariates. For the statistical test, the group affiliation is discarded in the restricted model. 


\section{Results}

275 when conducting group comparison in DT-MRI, which are: (i) is there a real added value of considering the whole tensor information as compared to a single scalar index or several scalar indices simultaneously ? (ii) is the GLM tensorbased method more effective than alternative tensor-based tests such as the

that $G L M-M D$ and $G L M-D T$ exhibit very close performance for the three first 

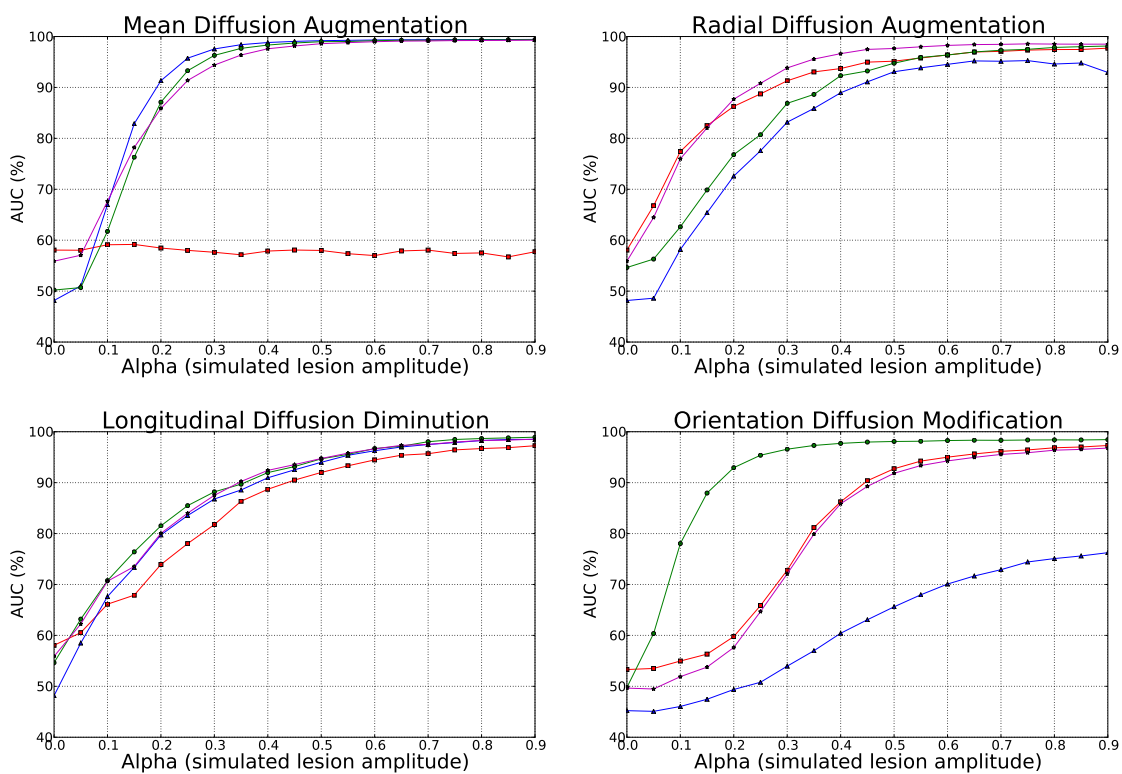

Figure 3: Comparison between the scalar-based methods ( $\mathbf{\square}=$ GLM-FA, $\boldsymbol{\Delta}=$ GLM-MD), the multivariate scalar-based method $(\star=$ GLM-FA + MD) and the Euclidean tensor-based $\operatorname{method}(\bullet=$ GLM-DT $)$.

kinds of lesions and that $G L M-F A$ and $G L M-F A+M D$ outperform the two other methods for detecting radial diffusion augmentation. For the modification of diffusion orientation, one would have expected that the three scalar-based methods lead to almost 50\% AUC (i.e., random detection) for all lesion amplitudes, since the change of orientation does not alter the tensor eigenvalues. The observed detections are explained by the Gaussian filtering, which is performed as a preprocessing step: averaging rotated tensors with neighboring non-rotated tensor produces tensors with different shapes, yielding changes in FA and MD. To conclude, the GLM-DT method seems to be the one that offers the best compromise for detecting all changes, although it does not clearly outperform the three other methods for all kinds of lesions. 

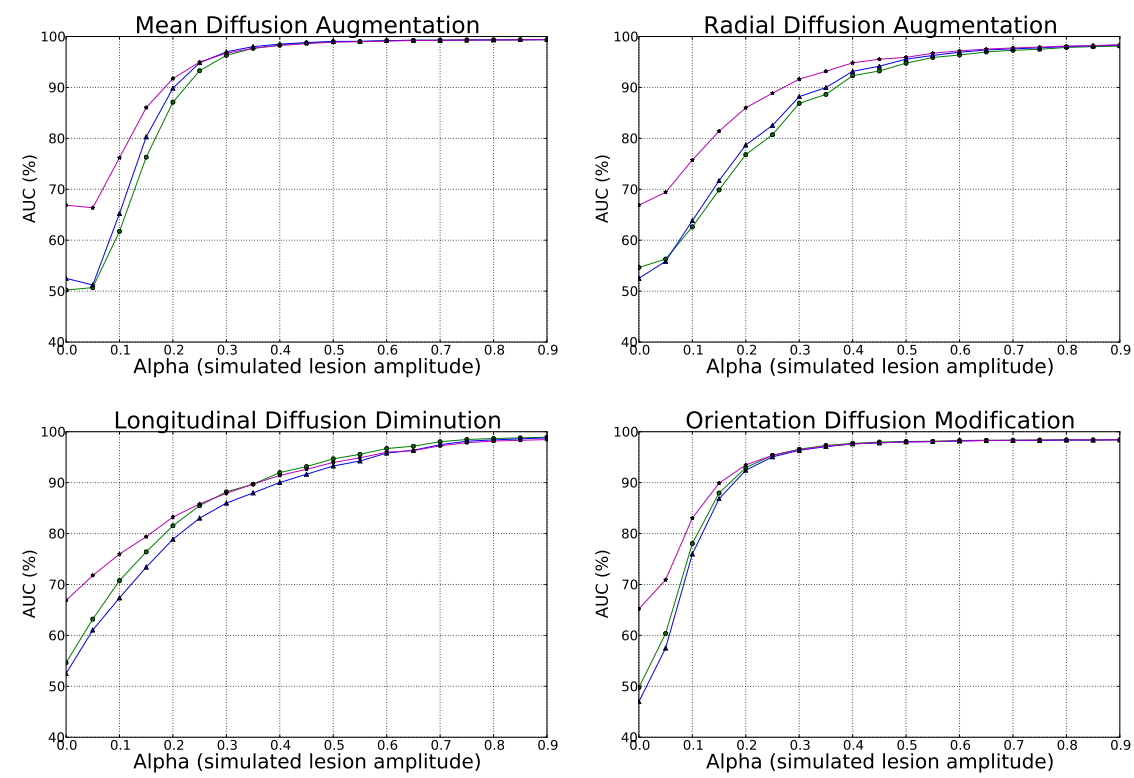

Figure 4: Comparison between the tensor-based GLM methods $(\bullet=$ GLM-DT with covariates, $\boldsymbol{\Delta}=$ GLM-DT without covariate) and the tensor-based Cramér test ( $\star=$ Cramér).

\subsubsection{GLM tensor-based regression vs tensor-based Cramér test}

Fig. 4 shows the results of the comparison between the GLM-DT and the Cramér test. Results of the GLM-DT without covariate are also presented in order to investigate the influence of considering the age as a covariate (remind that the Cramér test does not account for covariates). GLM-DT with and without covariate lead to very close results, while the Cramér test outperforms these two methods for the four kinds of simulated lesions, especially for small lesion amplitudes. Notice that the two groups of subjects used for the simulation study are very homogenous in age ( $29 \pm 5$ year old vs $27.1 \pm 3.8$ year old), explaining why there are little differences between GLM-DT with and without covariate. The Cramér test clearly exhibits the best statistical power, but its performance could have seriously been deteriorated if the populations were more heterogeneous in age. 

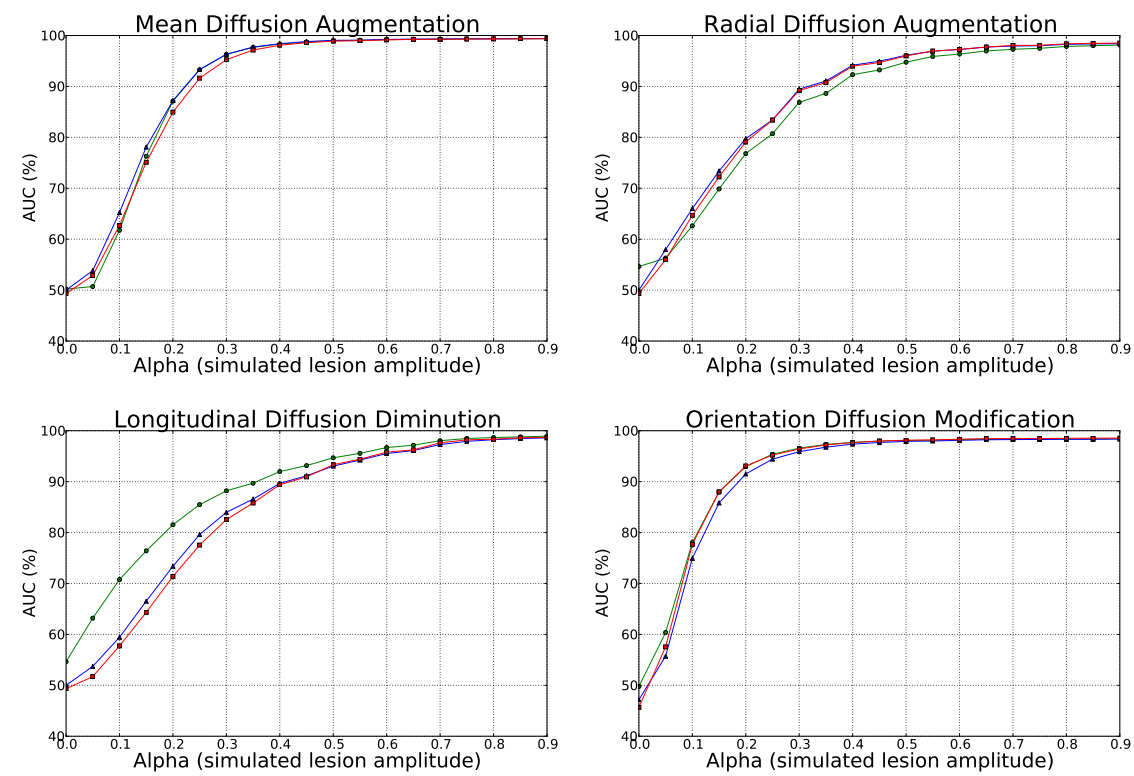

Figure 5: Influence of the manifold $(\bullet=$ GLM-DT, $\boldsymbol{\Delta}=$ GLM-LOG-DT, $\boldsymbol{\square}=$ MGLM-DT $)$.

\subsubsection{Manifold influence}

Fig. 5 sums up the results of the comparison between the Euclidean, the Log-Euclidean and the Riemannian manifolds. It highlights that the performance of the methods varies with the kind of simulated changes. GLM-DT outperforms the two other methods for detecting a longitudinal diffusion diminution. On the other hand, the GLM-LOG-DT and the MGLM-DT exhibit slightly better results for detecting an augmentation of the radial diffusion. Notice that all the three methods show similar performance for detecting either modifications of orientation or mean diffusion augmentation. Finally, it has to be pointed out that the Log-Euclidean and Riemannian manifold frameworks lead to close results.

\subsubsection{Characterization}

Table 3 presents the results of the characterization procedure applied to three of the simulated data sets $(\alpha=0.2, \alpha=0.5, \alpha=0.9)$. The statistical variations of $\mathrm{AD}, \mathrm{RD}$ and if required $\mathrm{FA}$ (only for the ambiguous cases) are reported for 
the whole ground truth area (i.e., the corpus callosum). The results show that the characterization procedure performs well for most cases. Two cases deserve a bit more explanation, namely the radial diffusion augmentation for $\alpha=0.9$ and the longitudinal diffusion diminution for $\alpha=0.5$ and 0.9 . Concerning the radial diffusion augmentation at $\alpha=0.9$, one would not have expected to observe a significant change for the $\mathrm{AD}$. It has to be pointed out that the pvalue associated with the $\mathrm{AD}$ modification is high $(p=0.023)$ as compared to the one associated to the RD index $\left(p=9.7410^{-11}\right)$, thus still objectifying that this is mainly a change of radial diffusion. In the same way, concerning the longitudinal diffusion diminution ( $\alpha=0.5$ and 0.9 ), one would not have expected to observe a significant change for the RD. It has to be pointed out that the $\mathrm{p}$-value associated with the RD modification is also high $(p=0.016$ for $\alpha=0.5$ and $p=0.019$ for $\alpha=0.9$ ) as compared to the one associated to the $\operatorname{AD}$ index $\left(p=1.310^{-11}\right.$ for $\alpha=0.5$ and $p=3.310^{-13}$ for $\alpha=0.9$ ). The fact that the AD is decreasing in a larger extent that the RD is also confirmed by the significant decrease of the FA. Consequently, according to Table 1, this modification will still be well categorised as a longitudinal diffusion diminution.

This phenomenon is in fact a side effect of the registration step. Indeed, if the registrations are estimated on the lesion-free images $(\alpha=0)$ and the corresponding transformations are applied on the images with simulated lesions, then the $\mathrm{AD}$ (resp. the $\mathrm{RD}$ ) become unaltered for the radial diffusion augmentation (resp. the longitudinal diminution). The reason why the registration causes these changes of $\mathrm{AD}$ (resp. $\mathrm{RD}$ ) is related to the large modification on the FA induced by the simulated lesion, the FA being precisely used to drive the registration process. Note that we do not observe in the real scenario such a modification of FA on such a large spatial extent, thus limiting the chance of getting such distortions in the estimated deformation fields.

\subsection{Results on $N M O$ patients}

In order to obtain comparable detection maps for all statistical analysis, p-value maps should first be computed. Unfortunately, this step can only be 


\begin{tabular}{|c|c|c|c|c|c|c|c|c|c|}
\hline \multirow[b]{3}{*}{ Simulated lesion } & \multicolumn{9}{|c|}{ Amplitude of the simulated lesion } \\
\hline & \multicolumn{3}{|c|}{0.2} & \multicolumn{3}{|c|}{0.5} & \multicolumn{3}{|c|}{0.9} \\
\hline & $\mathrm{AD}$ & $\mathrm{RD}$ & FA & $\mathrm{AD}$ & $\mathrm{RD}$ & FA & $\mathrm{AD}$ & $\mathrm{RD}$ & FA \\
\hline $\begin{array}{l}\text { Mean diffusion } \\
\text { augmentation }\end{array}$ & + & + & n.s. & + & + & n.s. & + & + & n.s. \\
\hline $\begin{array}{l}\text { Radial diffusion } \\
\text { augmentation }\end{array}$ & n.s. & + & & n.s. & + & & - & + & \\
\hline $\begin{array}{c}\text { Longitudinal } \\
\text { diffusion diminution }\end{array}$ & - & n.s. & & - & - & - & - & - & - \\
\hline $\begin{array}{c}\text { Diffusion orientation } \\
\text { modification }\end{array}$ & n.s. & n.s. & & n.s. & n.s. & & n.s. & n.s. & \\
\hline
\end{tabular}

Table 3: Results of the characterization procedure for the GLM-DT method (Legend: + = significant augmentation $(p<0.05),-=$ significant diminution $(p<0.05)$, n.s. $=$ not significant)

done through permutations for the MGLM and Cramér test, which would have been computationnaly prohibitive, especially for the MGLM method. In order to make a fair comparison, we chose to adjust the threshold for each method so that the same number of voxels are detected within the white matter mask. In all experiments presented in the sequel, statistical maps were thresholded in order to keep the $5 \%$ most significant voxels within the white matter mask; a cluster-based threshold of $N_{c}=10$ was then applied.

\subsubsection{Scalar-based vs tensor-based regression}

Fig. 6 shows the results obtained by the two univariate scalar-based methods (GLM-FA and GLM-MD), the multivariate scalar-based method (GLM$F A+M D)$ and the Euclidean tensor-based method (GLM-DT) for the comparison between 34 NMO patients and 22 healthy subjects.

Similarly as for the experiments on synthetic data (see section 4.1.1, the 


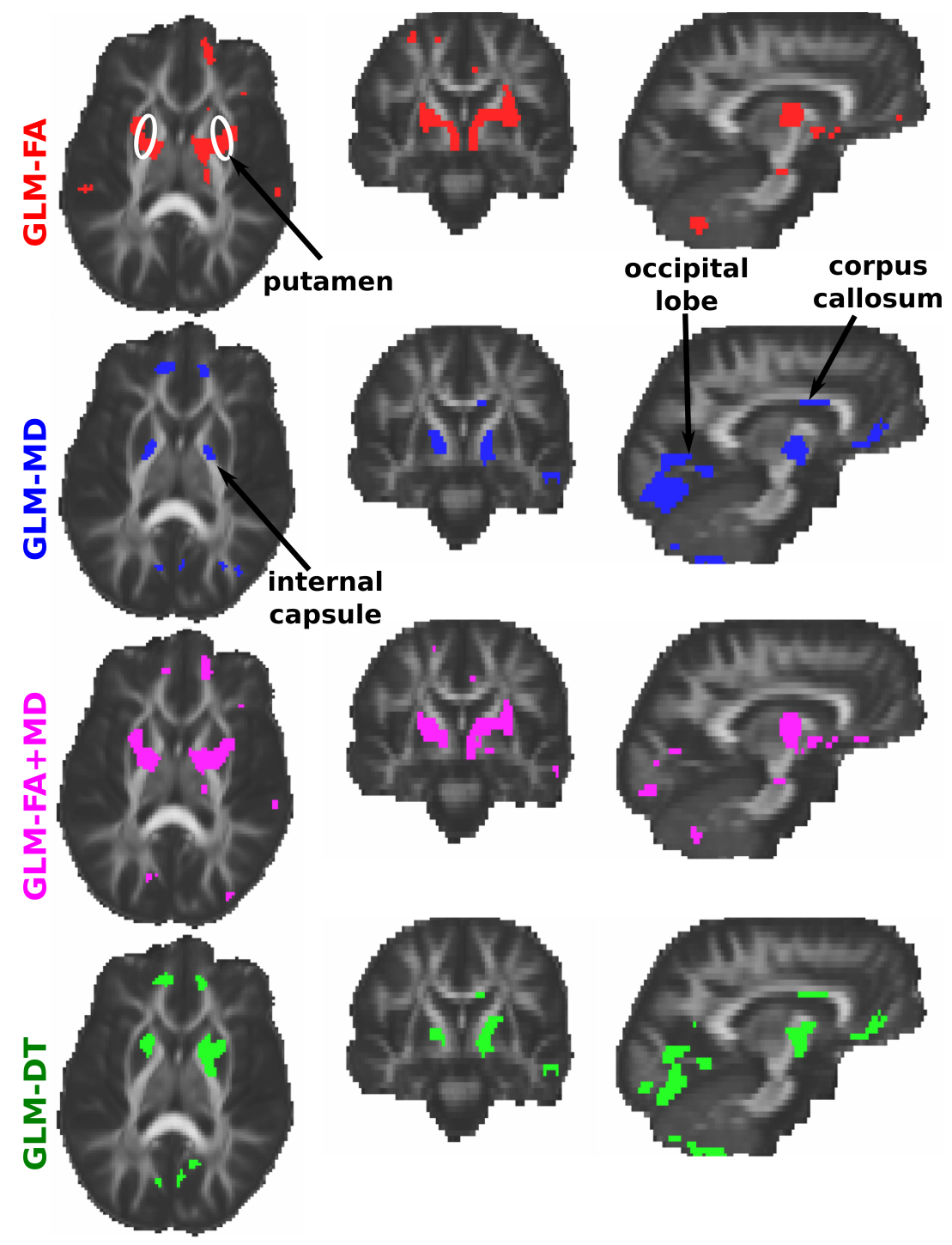

Figure 6: Comparison between the scalar-based methods (GLM-FA, GLM-MD and GLM$F A+M D)$ and the Euclidean tensor-based method (GLM-DT). Statistical maps were thresholded in order to keep the $5 \%$ most significant voxels within the white matter mask; a clusterbased threshold of $N_{c}=10$ was then applied. 


\subsubsection{GLM tensor-based regressions vs tensor-based Cramér test}

Fig. 7 shows the results of the comparison between the GLM-DT and the Cramér test. Results of the GLM-DT without covariate are also presented as well as the test evaluating the influence of the age using the GLM-DT with 

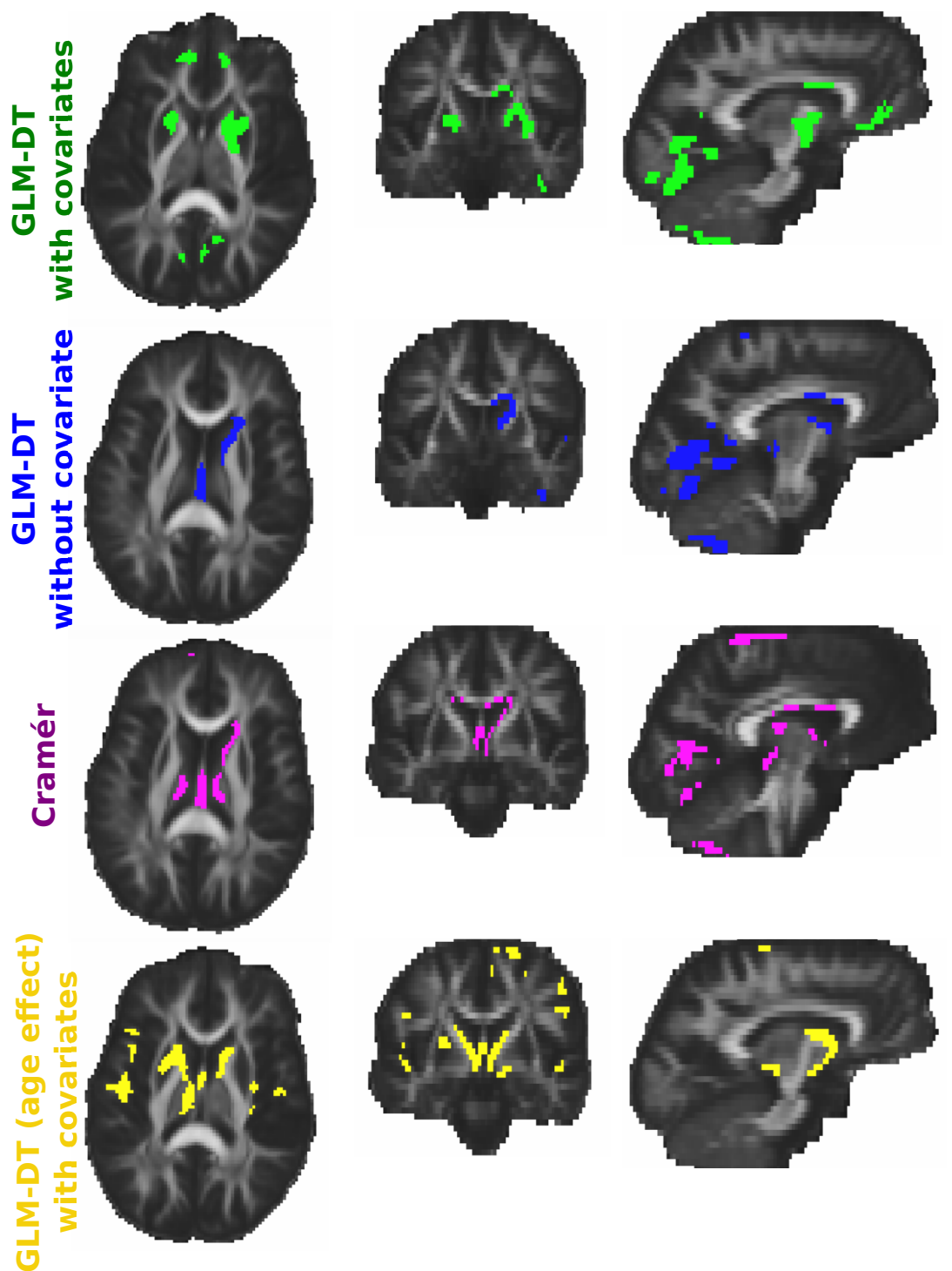

Figure 7: Comparison between the GLM tensor-based methods (GLM-DT with and without covariates) and the dedicated tensor-based test (Interpoints). Statistical maps were thresholded in order to keep the $5 \%$ most significant voxels within the white matter mask; a clusterbased threshold of $N_{c}=10$ was then applied. 
$D T$ with and without covariate lead to significantly different results, which is explained by the fact that the two populations under study are in that case not homogeneous in age (NMO: $44.9 \pm 12.2$ vs Controls: $28 \pm 4.4)$. Testing the effect of age using the GLM-DT with covariates (Fig. 7, bottom part) exhibit detections in regions around the ventricles and in several cortical areas. These detections are in fact the consequence of brain atrophy related to aging, which induces enlargement of brain ventricles and cortical sulci. Although the GLM-DT without covariate and the tensor-based Cramér test both succeed to detect keys regions of the NMO such as the occipital lobe, they also detect many regions around the ventricles related to age effect. Consequently, these results emphasize the importance of taking into account covariates in group study in order to bring out only the influence of a given effect.

\subsubsection{Manifold influence}

Fig. 8 sums up the results from the comparison between the Euclidean, the Log-Euclidean and the Riemannian manifolds. Visually, all three methods lead to very close results, which does not allow us to assert that one method outperforms the others. To strengthen this claim, the Dice coefficient between the detection maps obtained for each pair of methods is plotted in Fig. 9 for several statistical thresholds. This objectifies the good agreement between the tensor-based methods, with a Dice coefficient always greater than 0.7 . The best agreement is obtained between the GLM-LOG-DT and MGLM-DT frameworks (Dice coefficient greater than 0.9), which is in accordance with the conclusion drawn from simulated data.

\subsubsection{Characterization of detected regions}

The characterization procedure is applied on the detection map obtained with the GLM-DT method by considering a statistical threshold $p_{F D R}=0.05$ and cluster threshold $N_{c}=10$. It leads to the detection of nineteen significant clusters located in eigth different anatomical regions. The sign and the statistical 


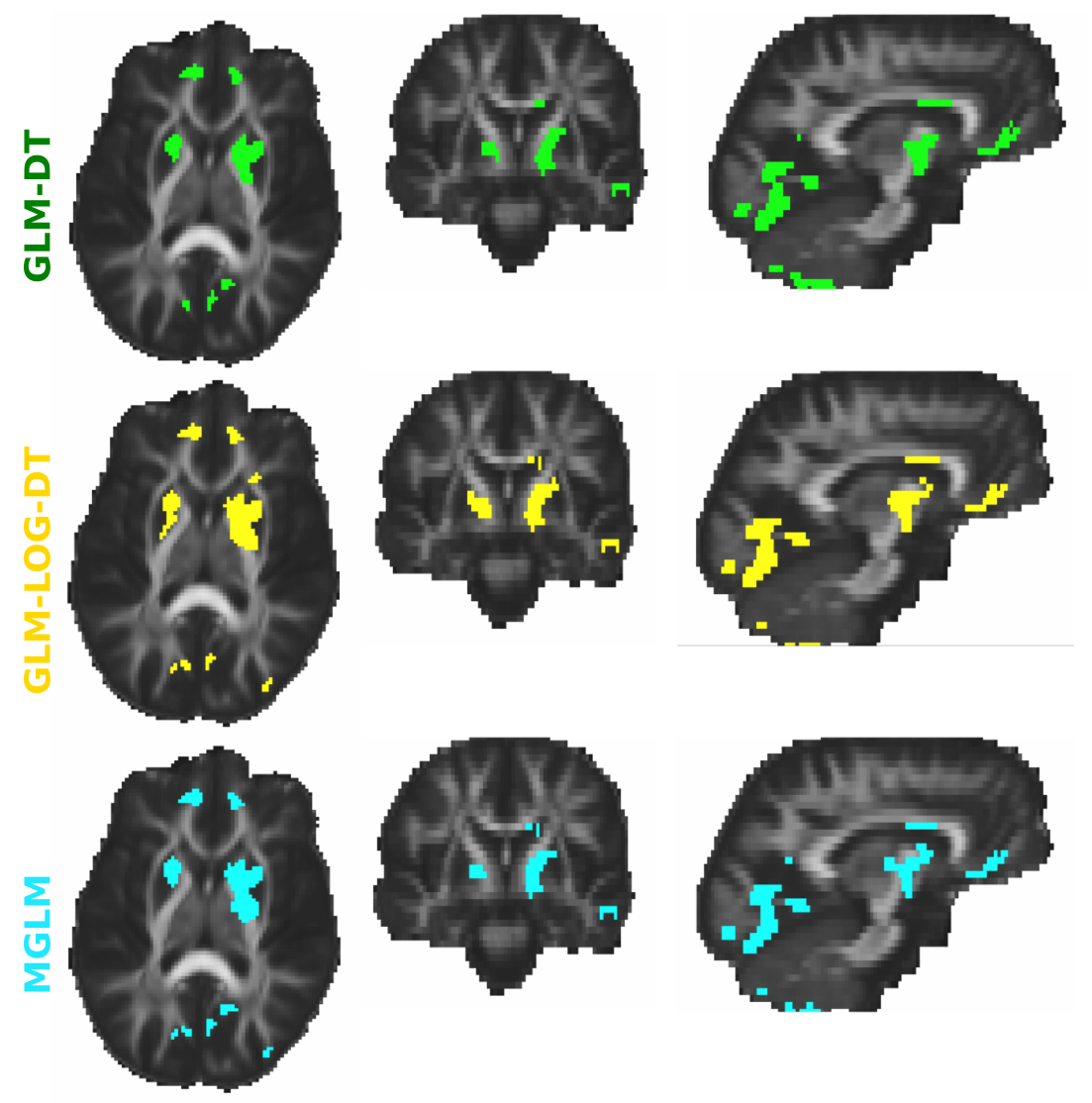

Figure 8: Influence of the manifold for conducting tensor-based comparison between NMO patients and healthy controls. Statistical maps were thresholded in order to keep the $5 \%$ most significant voxels within the white matter mask; a cluster-based threshold of $N_{c}=10$ was then applied. 


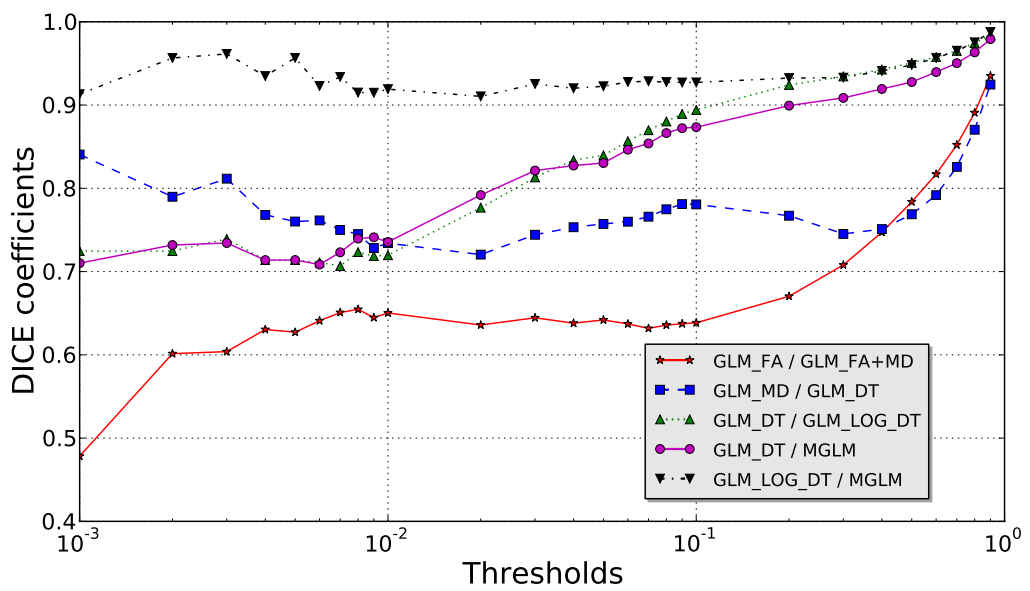

Figure 9: Dice coefficient between the detection maps obtained for every couples of methods $v s$ percentage of detected voxels in logarithmic scale. Only the couples of methods which have a Dice coefficient superior to 0.5 are displayed.

significance $(p<0.05)$ of the change for each scalar index (AD, RD and FA) are reported for each cluster in Table 4.

Among theses clusters, eigth are classified as deleterious changes, six as favorable changes and five as uncategorisable changes. Four clusters in the occipital lobe are characterized as an augmentation of the mean diffusivity in the patient group as compared to the control group, which may reflect a demyelination process that is probably related to the visual disorders induced by the pathology. Similar signature is observed for the cluster located in the corpus callosum. One cluster located in the parietal lobe presents a radial diffusion augmentation. Two other clusters located respectively in the frontal lobe and in the cerebellum present an axial diffusion diminution. All these changes can also be linked to a demyelination process.

The six clusters that are categorized as favorable changes might be the consequences of compensation phenomena or therapeutic drugs effects. The remaining clusters belong to the uncategorisable group, which can be explained by either a modification of diffusion orientation or because these clusters present 


\begin{tabular}{|c|c|c|c|c|c|}
\hline \multicolumn{2}{|c|}{ Brain location } & \multirow{2}{*}{$\begin{array}{c}\text { Impacted fiber tracts } \\
\text { optic radiation } \\
\text { inf. long. fasciculus } \\
\text { corpus callosum (splenium) }\end{array}$} & \multirow{2}{*}{$\begin{array}{c}\mathrm{AD} \\
+ \\
+\end{array}$} & \multirow{2}{*}{$\begin{array}{c}\text { RD } \\
+ \\
+\end{array}$} & \multirow{2}{*}{$\begin{array}{l}\text { FA } \\
\text { n.s. }\end{array}$} \\
\hline \multirow{4}{*}{ Occipital lobe } & r. \& 1. & & & & \\
\hline & 1. & $\begin{array}{c}\text { optic radiation } \\
\text { corpus callosum (splenium) }\end{array}$ & + & + & n.s. \\
\hline & r. & inf. fronto-occipital fasciculus & + & + & n.s. \\
\hline & r. & undefined & + & n.s. & \\
\hline Hippocampus & 1. & undefined & + & n.s. & \\
\hline Corpus callosum & r. & corpus callosum (body) & + & + & n.s. \\
\hline Parietal lobe & r. & sup. long. fasciculus & + & + & - \\
\hline \multirow{3}{*}{ Frontal lobe } & r. & $\begin{array}{l}\text { corpus callosum (genu) } \\
\text { inf. fronto-occipital }\end{array}$ & n.s. & n.s. & \\
\hline & 1. & inf. fronto-occipital & - & - & n.s. \\
\hline & 1. & corpus callosum (genu) & - & n.s. & \\
\hline \multirow[t]{2}{*}{ Internal capsule } & r. & $\begin{array}{c}\text { inf. fronto-occipital } \\
\text { dentate-rubro-thalamo cortical }\end{array}$ & n.s. & n.s. & \\
\hline & r. & cortico-spinal & - & - & n.s. \\
\hline \multirow{2}{*}{ Temporal lobe } & r. & arcuate fasciculus & n.s. & n.s. & \\
\hline & r. & inf. long. fasciculus & - & - & n.s. \\
\hline \multirow{4}{*}{ Cerebellum } & 1. & undefined & - & n.s. & \\
\hline & r. & undefined & - & - & n.s. \\
\hline & 1. & undefined & n.s. & n.s. & \\
\hline & r. & undefined & n.s. & n.s. & \\
\hline
\end{tabular}

Table 4: Results $\left(p_{\text {corrected }}<0.05\right)$ of the characterization procedure for the GLM-DT method (statistical threshold $p_{F D R}=0.05$ and cluster threshold $N_{c}=10$ ). (Legend: $+=$ significant augmentation, $-=$ significant diminution, n.s. $=$ not significant, r. $=$ right, $\mathrm{l} ;=$ left, inf. = anterior, sup. = superior, long. = longitudinal) 

of the scalar indices.

We are well aware that there are multiple other options to perform such a classification. We chose this straighforward and computationally efficient way in order to keep the same statistical framework as for the detection method using GLM with the same covariates.

\section{Discussion}

\subsection{Scalar-based vs tensor-based regression}

A previous study has already compared scalar-based $v s$ several tensor-based methods for group comparison in DT-MRI [6]. They have considered both Euclidean and Log-Euclidean metrics. One of the main difference with our study is that the authors did not consider any covariates such as age or gender in their statistical analysis. They also did not have access to a Riemannian Manifold regression method such as 9]. The comparison between FA-based and tensorbased methods was also conducted on both simulated and real data. The evaluation on simulated data highlights unsurprisingly that tensor-based methods are the only ones that are able to detect modification of diffusion orientation. The conclusion concerning the detection of FA alterations is less obvious, since the FA-based approach slightly outperforms the tensor-based method relying on the Hoteling's $T^{2}$ test, but exhibits a lower statistical power than the tensor-based method relying on multivariate permutation test. Our results are consistent with this paper since we also conclude that the tensor-based method is the only one that can efficiently detect every kinds of changes, although it can perform slightly worse than scalar-based methods for some particular changes (e.g. FAbased method best detects an augmentation of radial diffusion while MD-based method best detects an augmentation of mean diffusion). Their conclusions on a cohort of patients with a history of migraine is also not clear-cut since both FA-based and tensor-based methods can detect a cluster into the bank of the postcentral gyrus, but with a slightly greater spatial extent for the tensor-based 
method. This may be probably explained by a gain in statistical power caused

The tensor-based Cramér test has been previously reported as the best performer among various tensor-based statistical tests [6]. It has to be noticed that none of the methods compared in [6] allows to take into account explanatory

In the sight of our conclusions and the ones obtained by Whitcher et al [], we can give the following recommendation to help the reader to choose between conducting a group study using either a scalar-based or a tensor-based method. If one has a strong a priori about the kind of changes to detect (e.g. a modification of the mean diffusivity), it is preferable to resort to the scalar-based approach on the appropriate scalar images (e.g. MD). This would be easier to implement and may lead to better performance. Moreover, this would avoid to resort to tensor-based dedicated methods for interpolation, smoothing and warping, which are not necessarily provided in standard neuroimaging libraries such as SPM or FSL. If one has no prior assumption on the expected changes, it would be preferable to use the tensor-based approach rather than performing several scalar-based analysis on different indices. This avoids the problem of multiple comparison (as compared to perform several tests on different indices) and leads to a unique detection map. Nonetheless, using a tensor-based method would necessarily require a characterization step (section 2.5) in order to help physicians or neuroscientists in their interpretation. Finally, one of the main added value of the tensor-based method is its ability to detect the modifications of diffusion orientation. However, this may be of limited interest in the context of voxelwise group comparison since most pathologies do not cause modifications of diffusion orientation in a consistent area across a population. Modifications of orientation are generally observed in the vicinity of multiple sclerosis lesions or glioma and are generally related to the effect of oedema. Consequently, this property may be more interesting for the follow-up of a given patient 26] than for group studies.

\subsection{GLM tensor-based regression vs tensor-based Cramér test}


variables such as age. This was not a crucial point for their experiment on real

data since it involved populations of young adults with little age differences. Our experiments on simulated data also highlight the superiority in term of statistical power of the Cramér test over the GLM tensor-based regression. This is explained by the fact that these experiments also involve young adults of similar age. The conclusion obtained on the real database is drastically different, showing the superiority of the GLM tensor-based regression as compared to the Cramér test. This is explained by the age heterogeneity of the two populations under study. Taking into account covariates in neuroimaging studies appears to be a crucial point, especially for studies involving elderly subjects, since aging induces several brain modifications in terms of both atrophy and diffusion property alteration [27]. Consequently, if one has a strong a priori that there is no confounding variable that can hamper a group comparison, then a nonparametric statistical test such as the Cramér test should be considered. Otherwise, a GLM tensor-based regression should be preferred.

\subsection{Manifold influence}

In the literature, a few works have already evaluated the impact of the Euclidean, Log-Euclidean and affine invariant-Riemannian metrics for various images processing problems [11, 28] but there is still no consensus on the most appropriate manifold for handling diffusion tensors, especially in the context of group comparison. Some papers advocate the use of Log-Euclidean or Riemannian metrics for conducting DT-MRI group comparison [7, 5, 8, 9] while other papers claim that these metrics are not superior [6] or even are inferior [28] to the Euclidean one. In their comparison between Euclidean and Log-Euclidean metrics, Whitcher et al [6] conclude that there is no reason to prefer the LogEuclidean distance and that applying matrix logarithms to diffusion tensors tends to reduce the group difference observed in Euclidean space. Moreover, both theoretic analysis and experimental results reported in 28] support the claim that the Euclidean metric is more appropriate than an affine-invariant metric for the analysis of diffusion coefficients and tensors. 


\begin{tabular}{|c|c|}
\hline Methods & Computation time $(s)$ \\
\hline GLM-FA & 135 \\
GLM-MD & 135 \\
GLM-DT & 135 \\
GLM-LOG-DT & 148 \\
MGLM-DT & 253392 \\
\hline
\end{tabular}

Table 5: Computing time for conducting the statistical analysis on the NMO database.

Our experimental results on simulated lesions highlight that all metrics can

Log-Euclidean approaches.

In conclusion, this study suggests that, despite the mathematical elegance offered by the Riemannian framework, its superiority on both simulated and real clinical data could not be demonstrated compared to the Euclidean and present the advantage to be easily implementable and computationally effective. This conclusion is of course limited to the scope of the present application.

Finally, we want to discuss the fact that the Euclidean framework is gen-

\footnotetext{
$\sqrt{\text { https://www.nitrc.org/projects/riem_mglm }}$
} 
erally considered as a mathematically incorrect metric for handling diffusion

the difference between two positive definite matrices does not necessarily yield a positive definite matrix). However, positive definite matrices lie on a convex space. This means that a linear combination of positive definite matrices with positive coefficients will yield to a positive definite matrix. Consequently, comalso produce a valid positive definite matrix. Besides, computing the Euclidean distance between two positive definite matrices $D_{1}$ and $D_{2}$ amounts to measure the length of the straight line that link these two matrices. This makes sense since all matrices $D$ that belong to this line $\left(D=\lambda D_{1}+(1-\lambda) D_{2}, \lambda \in[0,1]\right)$ inappropriate to handle diffusion tensor, thanks to the convexity property.

One problem that can arise when conducting statistical analysis on tensors in the Euclidean space, is related to the use of the Gaussian distribution assumption. Indeed, it may lead to associate nonzero probabilities to invalid tensors. This can be an issue if the data lie close to the boundary of admissible space. Nonetheless, this side effect can be neglected if the data are far from this boundary, which is generally the case in the context of our application, in particular thanks to the gaussian smoothing of the data which is performed before conducting the statistical analysis. To illustrate this discussion, we can make a somewhat simplistic analogy with the positive real space $\mathbb{R}^{+}$, which is also a convex space but not a vector space. Although using an Euclidean metric on this space can be considered as mathematically incorrect, many people are still conducting student t-test to compare e.g. age, size or weight between populations.

\subsection{Limitations of the study}

Although this study was carefully prepared, our work still suffers from some limitations that are discussed in this section.

First, we have to point out that group study should ideally be conducted 
on isotropic data. Unfortunately, this was not the case for the experiments presented in this paper since we used data that came from a clinical scanner using standard sequence parameters optimised for the clinical routine. We acknowledge that this is a limitation of our study and that the results obtained on real data should be interpreted with caution. In fact, we do not really expect that resolution anisotropy will induce spurious false detection, but rather that it will introduce more variability in the data that might prevent detecting some relevant change. Spurious false detections might appear for instance if one group is more prone to head motion than the other, which is not the case in the context of this study.

Secondly, one can wonder whether the conclusions drawn from the simulation study are really relevant in the sight of the small number of subjects in each group, which would be considered too small in most neuroimaging studies. In real neuroimaging studies, the size of the population should generally be larger for two reasons: (i) the effect to be detected is generally small as compared to the population variability, thus requiring a large number of subjects to make it statistically significant; (ii) the statistical tests generally require a large number of subjects (generally $N>30$ ) to make the assumption on the statistics distribution valid. In our simulation study, we tried to get around these two points by (i) controling the effect size to make it sufficiently high as compared to the population variablity and by (ii) conducting the ROC curve analysis directly on the computed statistics and not on the corresponding p-values, which could have been wrongly estimated for such a small sample size.

Finally, it has to be noticed that we have conducted our investigation on the second-order tensor model, whose limitation to properly model fiber crossing is well known. Being aware of this limitation, we chose to focus our simulation experiment on the corpus callosum, which consists of strongly transverse white matter fibers tracts connecting the two hemispheres characterized by a single left-right orientation. Nevertheless, the conclusion drawn from the real data should be interpreted cautiously given the numerous fiber crossing areas in the brain. As a perspective, we plan to investigate more complex diffusion models, 
such as for instance high order tensor models [29].

\section{Conclusions and perspectives}

In this paper, we have investigated on both simulated and real data whether there is a real added value of considering the whole tensor information for conducting voxel-based group studies. To this end, we compared two statistical tests dedicated to tensor, namely the Cramér test and a tensor-based extension of the General Linear Model, the latter presenting the advantage to account for confounding variables. We have also evaluated the impact of different manifold for estimating the GLM parameters.

First, we concluded that if there is no prior assumption about the nature of the expected changes, it would be preferable to use the tensor-based approach rather than performing several scalar-based analyses on different indices. Conversely, if one has a strong a priori about the kind of expected change, it is preferable to resort to the scalar-based approach on the corresponding scalar image. We also want to point out that the tensor-based approach is relevant only if it is followed by a characterization step in order to help the physician or the neuroscientist to interpret the detected changes. Secondly, we concluded about the importance of including covariates in the statistical tests, especially for studies involving elderly subjects, since aging induces several brain modifications in terms of atrophy and diffusion property alteration. Nonetheless, if one has a strong a priori that there is no confounding variable that can hamper a group comparison, then a nonparametric statistical test such as the Cramér test should be considered. Finally, the evaluation of the impact of different manifolds (Euclidean, Log-Euclidean and affine-invariant Riemannian metrics) on group comparisons on tensor shows that all the methods perform well and lead to almost the same performance, with a significant computational overhead for the Riemannian framework. 


\section{Acknowledgment}

We would like to thank Pr Jérome de Sèze for the recruitment of NMO patients and Pr Stéphane Kremer for image acquisitions.

\section{References}

[1] M. Cercignani, Strategies for PatientControl Comparison of Diffusion MR Data, Oxford University Press, 2010.

[2] W. Penny, K. Friston, J. Ashburner, S. Kirbel, T. Nichols, Statistical Parametric Mapping: The Analysis of Functional Brain Images, Elsevier LTD, Oxford, 2006.

[3] S. Smith, M. Jenkinson, H. Johansen-Berg, D. Rueckert, T. Nichols, C. Mackay, K. Watkins, O. Ciccarelli, M. Cader, P. Matthews, T. Behrens, Tract based spatial statistics: voxelwise analysis of multi-subjects diffusion data, NeuroImage 31 (4) (2006) 1487-1505. doi:10.1016/j.neuroimage. 2006.02 .024

[4] M. Chapell, J. Brown, J. Dalrymple-Alford, A. Uluğ, R. Watts, Multivariate analysis of diffusion tensor imaging data improves the detection of microstructural damage in young professional boxers, Magnetic Resonance Imaging 26 (10) (2008) 1398-1405. doi:10.1016/j.mri.2008.04.004.

[5] A. Schwartzman, R. Dougherty, J. Taylor, Group comparison of eigenvalues and eigenvectors of diffusion tensors, Journal of the American Statistical Association 105 (490) (2010) 588-599. doi:10.1198/jasa.2010.ap07291

[6] B. Whitcher, J. J. Wisco, N. Hadjikhani, D. S. Tuch, Statistical group comparison of diffusion tensors via multivariate hypothesis testing, Magnetic resonance in medicine 57 (6) (2007) 1065-1074. doi:10.1002/mrm.21229. URL http://www.ncbi.nlm.nih.gov/pmc/articles/PMC3763074/ 
[7] H. Zhu, Y. Chen, J. Ibrahim, Y. Li, C. Hall, W. Lin, Intrinsic regression

a models for positive-definite matrices with applications to Diffusion Tensor Imaging, Journal of the American Statistical Association 104 (487) (2009) $1203-1212$. URL http://EconPapers .repec .org/RePEc : bes : jnlasa:v: 104: i : 487 : $\mathrm{y}: 2009: \mathrm{p}: 1203-1212$

¿ [8] Y. Yuan, H. Zhu, W. Lin, J. Marron, Local polynomial regression for symmetric positive definite matrices, Journal of the Royal Statistical Society 74 (4) (2012) 697-719. URL http://EconPapers.repec.org/RePEc:bla:jorssb:v:74:y:2012: $\mathrm{i}: 4: \mathrm{p}: 697-719$

[9] H. Kim, B. Bendlin, N. Adluru, M. Collins, M. Chung, S. Johnson, R. Davidson, V. Singh, Multivariate General Linear Models (MGLM) on Riemannian Manifolds with Applications to Statistical Analysis of Diffusion Weighted Images, in: Computer Vision and Pattern Recognition (CVPR), 2014 IEEE Conference on, 2014, pp. 2705-2712. doi:10.1109/CVPR. 2014. 352.

[10] A. Bouchon, V. Noblet, F. Heitz, J. Lamy, F. Blanc, J.-P. Armspach, General linear models for group studies in diffusion tensor imaging, in: Proceedings of International Symposium on Biomedical Imaging: From nano to macro, Beijing, China, 2014. doi:10.1109/ISBI.2014.6868100.

[11] V. Arsigny, P. Fillard, X. Pennec, N. Ayache, Log-euclidean metrics for fast and simple calculus on diffusion tensors, in: Magnetic Resonance in Medicine, 2006.

[12] X. Pennec, Probabilities and statistics on riemannian manifolds: Basic tools for geometric measurements, in: Proc. of Nonlinear Signal and Image Processing (NSIP'99), June 20-23, Antalya, Turkey, 1999, pp. 194-198.

[13] I. Oguz, M. Farzinfar, J. Matsui, F. Budin, Z. Liu, G. Gerig, H. Johnson, 
[14] H. Zhu, H. Zhang, J. G. Ibrahim, B. S. Peterson, Statistical analysis of diffusion tensors in diffusion-weighted magnetic resonance imaging data, Journal of the American Statistical Association 102 (480) (2007) 1085-

[18] T. Nichols, A. Holmes, Nonparametric permutation tests for functional neuroimaging: A primer with examples, Human Brain Mapping 15 (2002) 1-25. doi:10.1002/hbm.1058.

[19] H. Zhu, J. G. Ibrahim, N. Tang, D. B. Rowe, X. Hao, R. Bansal, B. S. in Neuroinformatics 8 (4). doi:10.3389/fninf.2014.00004. 1102.

URL http://www.jstor.org/stable/27639963

[15] D. K. Jones, M. R. Symms, M. Cercignani, R. J. Howard, The effect of filter size on $\{\mathrm{VBM}\}$ analyses of DT-MRI data, NeuroImage 26 (2) (2005) 546554. doi:http://dx.doi.org/10.1016/j.neuroimage.2005.02.013.

[16] V. Noblet, C. Heinrich, F. Heitz, J.-P. Armspach, Retrospective evaluation of a topology preserving non-rigid registration method, Medical Image Analysis 10 (3) (2006) 366-384. doi:10.1016/j.media.2006.01.001.

[17] D. Alexander, C. Pierpaoli, P. Basser, J. Gee, Spatial transformations of diffusion tensor magnetic resonance images, IEEE Transactions on Medical Imaging 20 (11) (2001) 1131-1139. doi:10.1109/42.963816.

Peterson, A statistical analysis of brain morphology using wild bootstrapping, IEEE Transactions on Medical Imaging 26 (7) (2007) 954-966. doi:10.1109/TMI.2007.897396.

[20] L. A. Harsan, P. Poulet, B. Guignard, J. Steibel, N. Parizel, P. de Sousa, N. Boehm, D. Grucker, M. S. Ghandour, Brain dysmyelination and recovery assessment by noninvasive in vivo diffusion tensor magnetic reso-

M. Styner, DTIPrep: quality control of diffusion-weighted images, Frontiers nance imaging, Journal of Neuroscience Research 83 (3) (2006) 392-402. doi:10.1002/jnr.20742. 
[21] H. Boisgontier, V. Noblet, F. Heitz, L. Rumbach, J.-P. Armspach, Generalized likelihood ratio tests for change detection in diffusion tensor images: application to multiple sclerosis, Medical Image Analysis 16 (1) (2012) 325338. doi:10.1016/j.media.2011.08.007.

[22] T. Fawcett, An introduction to ROC analysis, Pattern Recognition Letters 27 (8) (2006) 861-874. doi:10.1016/j.patrec.2005.10.010.

[23] C. Yu, F. Lin, K. Li, T. Jiang, W. Qin, H. Sun, P. Chan, Pathologenesis of normal-appearing white matter damage in neuromyelitis optica: Diffusion-

口 Tensor MR Imaging, Radiology 246 (1) (2008) 222-228. doi:10.1148/ radiol. 2461062075

[24] D.-D. Zhao, H.-Y. Zhou, Q.-Z. Wu, J. Liu, X.-Y. Chen, D. He, X.-F. He, W.-J. Han, Q.-Y. Gong, Diffusion tensor imaging characterization of occult brain damage in relapsing neuromyelitis optica using 3.0T magnetic resonance imaging techniques, NeuroImage 59 (4) (2012) 3173-3177. doi:10.1016/j.neuroimage.2011.11.022

[25] F. C. R. Lopes, T. Doring, C. Martins, F. C. Cabral, F. R. Malfetano, V. C.

n. S. R. Pereira, S. Alves-Leon, E. L. Gasparetto, The Role of Demyelination in Neuromyelitis Optica Damage: Diffusion-Tensor MR Imaging Study, Radiology 263 (1) (2012) 235-242. doi:10.1148/radiol.12111241 URL http://dx.doi.org/10.1148/radiol.12111241

[26] A. Grigis, V. Noblet, F. Heitz, F. Blanc, J. De Sèze, S. Kremer, L. Rumbach, J.-P. Armspach, Longitudinal change detection in diffusion MRI using multivariate statistial testing on tensors, NeuroImage 60 (2012) 2206-2221. doi:10.1016/j.neuroimage.2012.02.049

[27] F. M. Gunning-Dixon, A. M. Brickman, J. C. Cheng, G. S. Alexopoulos, Aging of cerebral white matter: a review of MRI findings, Int J Geriatr Psychiatry 24 (2) (2009) 109-117. 
765

[28] O. Pasternak, N. Sochen, P. J. Basser, The effect of metric selection on the analysis of diffusion tensor MRI data, NeuroImage 49 (3) (2010) 2190-2204. doi:10.1016/j.neuroimage.2009.10.071

[29] T. Gkamas, F. Renard, C. Heinrich, S. Kremer, A fourth order tensor statistical model for diffusion weighted MRI - application to population comparison, in: International Conference on Pattern Recognition Applications and Methods (ICPRAM), 2015, pp. 277-282. 